\title{
Assessing the Patient with Abdominal Obesity: Metabolic and Nutritional Factors
}

\author{
Pop Raluca1,2, Pop $\mathrm{M}^{1,3}$, Dogaru $\mathrm{G}^{4,5}$ \\ 1 PhD Student, University of Medicine and Pharmacy, Tîrgu Mureș, Romania \\ 2 Endocrinology Outpatient Clinic, County Emergency Clinical Hospital, Tîrgu Mureș, Romania \\ 3 Radiology Department, County Emergency Clinical Hospital, Tîrgu Mureș, Romania \\ ${ }^{4}$ Internal Medicine Department, University of Medicine and Pharmacy, Tîrgu Mureș, Romania \\ ${ }^{5}$ Nephrology Department, County Emergency Clinical Hospital, Tîrgu Mureș, Romania
}

Background: Abdominal obesity is a confirmed cardio-vascular risk factor and the elements influencing it are subject for research and intervention. The available nutritional evaluation methods are time consuming, subjective and a standardized approach is missing.

Aim: Standardized evaluation of patients with abdominal obesity.

Material and methods: Cross-sectional study on a convenience sample of 85 subjects who presented in the Endocrinology Outpatient Clinic of the Emergency Mures County Hospital between February - April 2013. Variables: age, sex, environment, BMI, waist, blood glucose, triglycerides, HDL-Cholesterol, blood pressure. Each patient filled a food frequency questionnaire.

Results: None of the subjects had a "normal" food pyramid. Sex has no influence on the food pyramid. The meat and protein food group is the only one significantly influenced by the environment $(p=0.04)$. Patients with dyslipidemia consume lower amounts of sweets (1.13 vs. 1.83 servings, $p=0.007$ ). Patients requiring metabolic treatment have significant higher waist values (101.32 vs. $93.07 \mathrm{~cm}, p=0.03)$. Patients with simple abdominal obesity consume significant lower amounts of meat and protein and higher amounts of fruit and vegetables.

Conclusions: A standardized approach to the patient with abdominal obesity using nutritional assessment tools and metabolic evaluation helps to identify those at risk and to give more tailored recommendations.

Keywords: abdominal obesity, food pyramid, nutritional assessment

Received: 13 July 2013

\section{Introduction}

Obesity is a well-recognized public health issue and there are world-wide intervention task-forces designed for its prevention and control [1]. In recent years, abdominal obesity has become the more concerning cardio-vascular risk factor to be taken into account and various studies were developed for better understanding of its pathophysiology [2,3]. Strategies designed for the management of this health problem have yet to be standardized.

The nutritional evaluation has to be a part of every general consultation, but it is time consuming and difficult to accomplish. Various tools are available [4,5] but none of them are fail-proof and it is recommended to use 2 or more for a complete image. Food frequency questionnaires are one of the best available methods for nutritional evaluation and they are used in national surveys as well [6].

Although there is much discussion about the visceral fat and its surrogate measurement, waist circumference [7], there is still no available guide for the evaluation of a patient with this condition.

So there are some questions left unanswered: is abdominal obesity to be taken into account in any patient? Should it be considered a diagnosis and should there be specific lab analysis recommended? Is the food frequency question-

Correspondence to: Marian Pop

E-mail: marian.pop@umftgm.ro naire a good enough nutritional tool? Our study was designed to try to respond to these questions. Its main objective was to propose a standardized approach to the patient with abdominal obesity.

\section{Methods}

\section{Subjects and study design}

The type of study was cross-sectional. The target population was represented by the patients with abdominal obesity. We used a convenience sample of 85 adult patients who presented themselves in the Endocrinology Outpatient Clinic of the Emergency Mures County Hospital between February and April 2013. The inclusion criteria were abdominal obesity defined according to the International $\mathrm{Di}$ abetes Federation (2006) and the willingness to participate by signing a written consent form. The exclusion criteria were coexisting psychiatric conditions that render the patient unable to fill the food frequency questionnaire (FFQ), other causes of increased waist circumference (pregnancy, congestive heart failure, cirrhosis, other edematous states) and the formal refusal of filling in the FFQ.

\section{Variables}

The variables analyzed were: age, sex, environment, blood pressure, serum lipid profile (total cholesterol, HDL cholesterol, and triglycerides), blood glucose, and food pyra- 
Table I. The association between serum metabolic profile and sweets consumption

\begin{tabular}{lccc}
\hline Serum measurement & $\begin{array}{c}\text { Relative risk } \\
\text { (RR) }\end{array}$ & $\begin{array}{c}\text { Confidence } \\
\text { interval (Cl) }\end{array}$ & p value \\
\hline Triglycerides & 0.38 & $0.16-0.87$ & 0.03 \\
Total cholesterol & 2.25 & $1.13-4.45$ & 0.02 \\
HDL cholesterol & 2.72 & $0.87-8.49$ & 0.08 \\
Blood glucose & 0.62 & $0.24-1.56$ & 0.34 \\
\hline
\end{tabular}

mid. All serum measurements were performed using standardized methods. The cut-off levels were defined according to the international guidelines available [8,9]. All patients filled a food frequency questionnaire with 129 items based on the one used in the National Health and Nutrition Examination Survey [6]. A web-based application was used for constructing the food pyramid [10].

\section{Data analysis}

The continuous variables were expressed as mean \pm standard deviation. Grubbs test was used for identifying outliers, only for the food frequency questionnaire. The difference in means in quantitative variables has been analyzed using the $\mathrm{t}$ student test or ANOVA for independent samples. Pearson's or Spearman correlation coefficients were used to estimate the relationship between the quantitative variables, while the chi-square test was used to associate the qualitative variables.

The statistical analysis was performed using MO Excel and GraphPad Prism 5. A value of $\mathrm{p}<0.05$ was considered statistically significant.

\section{Ethical considerations}

All patients signed a written consent; the study was approved by the ethical committee of the County Emergency Clinical Hospital of Tîrgu Mureș.

\section{Results}

From the original sample of 85 patients one was excluded due to missing lab results. The remaining sample had the following general characteristics: sex repartition -72 females and 12 men (85.71\% vs. $14.28 \%)$ with a mean age of $51.55 \pm 11.49$ years. $69.04 \%$ came from urban areas and $30.95 \%$ from the rural areas. 43 subjects $(53.19 \%)$ fulfilled the criteria for metabolic syndrome diagnosis, according to the International Diabetes Federation definition.

Sex had no significant influence on the food pyramid. Patients from rural areas consumed significant higher amounts of meat and protein than the ones from urban areas $(3.23$ vs. 2.42 servings/day, $\mathrm{p}=0.04)$. There is a weak, but significant negative correlation between age and consumption of concentrated sweets and saturated fat $(\mathrm{r}$ $=-0.28$, CI 95\% -0.47- $-0.08, \mathrm{p}=0.008)$. From the six food groups only the concentrated sweets and fat were associated with higher waist circumference (104.3 vs. 97.2, $\mathrm{p}=0.005)$. Patients having comorbidities associated that

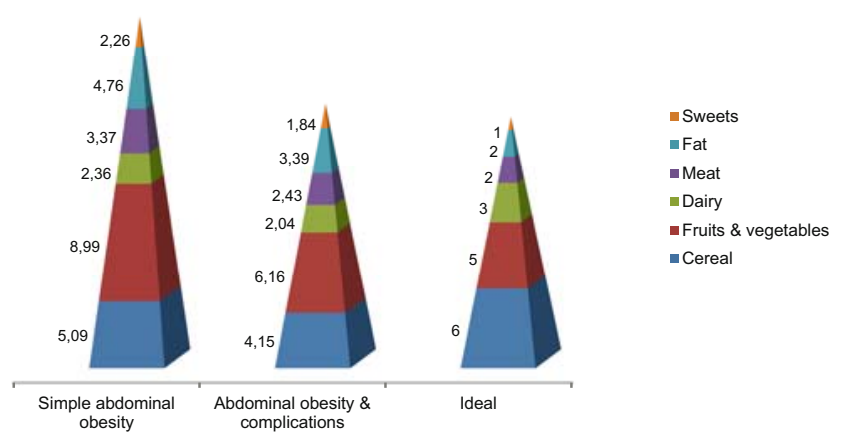

Fig. 1. The food pyramid

require treatment have significant higher waist circumference than the "healthy ones" 104.5 vs. 94.37, $\mathrm{p}=0.003$ ).

We found no association between waist circumference and the metabolic serum profile. We stratified the abdominal obesity into two groups: moderate risk (females 80 $87.9 \mathrm{~cm}$, males $94-101.9 \mathrm{~cm}$ ) and high risk (females $>88 \mathrm{~cm}$ and males $>102 \mathrm{~cm}$ ) and found that the higher the waist circumference, the lower the number of sweets servings.

Food pyramid - none of the subjects had a "normal" food pyramid, according to the 2011 recommendations. Patients consuming fewer servings of cereal and grains had significant lower serum triglycerides. (109.9 vs. $176.6 \mathrm{mg} /$ $\mathrm{dl}, \mathrm{p}=0.03$ ), while those consuming concentrated sweets and fat in higher amounts had significantly higher serum triglycerides ( 165.4 vs. $109.9 \mathrm{mg} / \mathrm{dl}, \mathrm{p}=0.01$ ). After analyzing each food group and its associations with the metabolic profile, we found that only the concentrated sweets food group has significant influence on the lipid profile, but not on the blood glucose (Table I).

As expected, diabetic patients consume significant lower amounts of sweets than the non-diabetic subjects ( 0.93 vs. $1.71, \mathrm{p}=0.01)$. Also, patients requiring treatment consume lower amounts from all food groups, significant only for the meat and protein (2.29 vs. 3.07, $\mathrm{p}=0.03)$.

Only $15.47 \%$ of subjects had simple abdominal obesity, $55.95 \%$ were already being treated for one or more metabolic disturbances and $28.57 \%$ were newly discovered patients. Subjects with simple abdominal obesity consume significant lower amounts of meat and protein (2.43 vs. 3.37 servings/day, $\mathrm{p}=0.03$ ) and higher amounts of fruits and vegetables ( 8.99 vs. 6.16 servings $/$ day, $p=0.006$ ). Figure 1 shows the food pyramid for the two groups, compared with the one considered ideal.

When comparing the subjects with metabolic syndrome with the others, we found no significant differences considering each food group independently, but after combining the sweets and fat groups, we found a significant association with a linear trend $(\mathrm{p}=0.01)$.

\section{Discussions}

In this study we analyzed abdominal obesity in relation with metabolic and nutritional factors. A recent meta-analysis [11] has shown that waist circumference measurement 
should be a part of general examination and that it helps in assessing the obesity-related mortality risk. Also the abdominal obesity is a well confirmed cardio-vascular risk factor $[7,12]$. We used a standardized approach that allows the diagnosis of multiple metabolic complications and the description of dietary habits. Our sample was a "young" one, fact that underlines the importance of searching for other risk factors in the presence of abdominal obesity. Multiple studies have addressed the issue of abdominal obesity, but there is still no consensus whether we should screen everybody for this condition or not $[7,3]$.

It is important to emphasize that more than a quarter of the sample were not previously diagnosed with metabolic disturbances and did not receive appropriate management. Only a small percentage of our sample had only abdominal obesity as a risk factor and more than a half fulfilled the metabolic syndrome criteria. Although other studies have demonstrated it $[13,14,15]$, we found no association between abdominal obesity and other risk factors, even after adjusting for sex or the environment. This is probably due to the convenience small sample used.

Nutritional assessment tools are "in fashion" these days and they are available both on- and offline [16,17]. Almost all papers studied agree that there is no perfect method in assessing dietary habits, all being subjective $[4,18,19]$. We chose a food frequency questionnaire, because in our opinion it gives a fair image of dietary habits for a bigger period of time than the $24 \mathrm{~h}$ recall. We constructed it based on the one used in the National Health and Nutrition Examination Survey [6] and we adapted it for national dietary patterns. Although it is a little bit time consuming, the subjects did not complain about this issue, but they found it to be difficult to think in servings and thus, maybe there is a little overestimation in their responses. We must underline the fact that in our knowledge this is the first nutritional tool developed specific for our population. It also gives the opportunity to construct the personal food pyramid which gives a good image of a person's diet.

We found no correlations between abdominal obesity and the serum profile, but our sample size was small. After constructing two groups of abdominal obesity we found a linear trend [20], the higher the waist, the lower the number of sweets servings. That means that obese patients try to control their diet, or that our subjects reported lower amounts, knowing that this is the recommended trend.

The concentrated sweets and saturated fat food group has the highest influence on the different parameters we studied. It is associated with higher waist circumference; it is weakly correlated with age - elderly patients consume lower amounts; it influencing the lipid profile, with strong impact on the cholesterol. The fact that the diabetic population in our study proved to consume significant lower amounts of sweets confirms the food frequency questionnaire as a tool that reflects the dietary habits of a subject. Also, patients that required treatment consumed lower amounts from each food group and had higher waist circumference.
We tried to analyze the dietary habits of people having simple abdominal obesity with no additional risk factor and found that they consume significant lower amounts of meat and higher amounts of fruit and vegetables, thus confirming that the latter is an important protecting factor against metabolic imbalances.

We must underline the limitations of our study. The sample size is small and the gender distribution doesn't follow the one in the general population. The serum measurements were not performed in the same lab, this factor beings a source of error. The food frequency questionnaire is subjected to errors, due to overestimation or memory bias.

\section{Conclusions}

The standardized approach proposed helps to identify patients at risk and the nutritional tool helps in constructing a better recommendation, with visual help (personal food pyramid), allowing more tailored advice.

\section{Acknowledgement}

This paper is partly supported by the Sectorial Operational Program Human Resources Development (SOP HRD), financed from the European Social Fund and by the Romanian Government under the contract number POSDRU 80641. Project partly supported through an Internal Research Grant of the University of Medicine and Pharmacy of Tîrgu Mureș.

\section{References}

1. Ivanisević P, Bojić L, Tomić S, et al. Clinico-epidemiological analysis of choroidal melanoma in Split Area, Croatia. Acta Med Croatica. 2011;65:257-261.

2. Uysal IÖ, Misir M, Polat K, et al. Primary malignant melanoma of the nasal cavity. J Craniofac Surg. 2012;23:2-5.

3. Yamamura K, Kondo K, Moritani S. Primary malignant melanoma of the stomach: report of a case. Surg Today. 2012;42:195-199.

4. Díaz-Sánchez A, Lara MÁ, Ortega P, et al. Colorectal melanoma: review of two distinct forms of presentation. Gastroenterol Hepatol. 2011;34:83-88.

5. Ramos JA, Ramos WE, Ramos CV. Melanoma of the female urethra. Indian J Urol. 2011;27:448-450.

6. Doublali M, Chouaib A, Khallouk A, et al. Primary malignant melanoma of prostate. Urol Ann. 2010;2:76-77.

7. Inoue M, Ishioka J, Kageyama Y, Fukuda H, Higashi Y. Primary malignant melanoma of the male urethra: a case report. Hinyokika Kiyo. 2008:54:305-308

8. Sosman JA, Kim KB, Schuchter L, et al. Survival in BRAF V600mutant advanced melanoma treated with vemurafenib. $\mathrm{N}$ Engl $\mathrm{J}$ Med. 2012;366:707-714.

9. Gandini S, Sera F, Cattaruzza MS, et al. Meta-analysis of risk factors for cutaneous melanoma: III. Family history, actinic damage and phenotypic factors. Eur J Cancer. 2005;41:2040-2059.

10. Anger M, Friedhofer H, Fukutaki MF, Ferreira MC, Landman G. Primary cutaneous melanoma: an 18-year study. Clinics (Sao Paulo). 2010;65:257263.

11. Döme B, Hendrix JC, Paku S et al. Biological perspectives. Alternative vascularization mechanism in cancer. Pathology and therapeutic implications. Am J Pathol 2007;170:1-15.

12. Battisti R, Nunes DH, Weber AL, Schweitzer LC, Sgrot I. Evaluation of the epidemiological profile and the mortality rate of the patients with primary cutaneous melanoma in Florianopolis - SC, Brazil. An Bras Dermatol. 2009;84:335-342.

13. Lugowska I, Kowalska M, Zdzienicki M, et al. The prognostic role of clinical factors, VEGF, IL-8 and sTNF-R1 in cutaneous melanomas at locoregional stage. Pol Merkur Lekarski. 2012;32:22-27. 
14. Garbe C, Hauschild A, Volkenandt M, et al. Evidence and interdisciplinary consense-based German guidelines: diagnosis and surveillance of melanoma. Melanoma Res. 2007;17:393-399.

15. Eggermont AM, Spatz A, Lazar V, Robert C. Is ulceration in cutaneous melanoma just a prognostic and predictive factor or is ulcerated melanoma a distinct biologic entity? Curr Opin Oncol. 2012;24:137-140.

16. Kovacević P, Visnjić M, Vlajković M, Kovacević T, Visnjić A. Sentinel node biopsy for skin melanoma. Vojnosanit Pregl. 2009;66:657-662.

17. Ohata C, Tadokoro T, Itami S. Expression of estrogen receptor beta in normal skin, melanocytic nevi and malignant melanomas. J Dermatol. 2008;35:215-221.
18. de Giorgi V, Gori A, Grazzini M, et al. Estrogens, estrogen receptors and melanoma. Expert Rev Anticancer Ther. 2011;11:739-747.

19. Vaisanen A, Kuvaja P, Kallioinen M, et al. A prognostic index in skin melanoma through the combination of matrix metalloproteinase-2, Ki67, and p53. Hum Pathol. 2011;42:1103-1111.

20. Helfrich I, Schadendorf D. Blood vessel maturation, vascular phenotype and angiogenic potential in malignant melanoma: One step forward for overcoming anti-angiogenic drug resistance? Mol Oncol. 2011;5:137149.

21. Mahabeleshwar GH, Byzova TV. Angiogenesis in Melanoma. Semin Oncol. 2007;34:555-565. 